\title{
Hepatic Hippo signaling inhibits development of hepatocellular carcinoma
}

\author{
Yuchen Liu', Xiaohui Wang', and Yingzi Yang ${ }^{1,2,3}$ \\ 'Department of Developmental Biology, Harvard School of Dental Medicine, Boston, MA; ${ }^{2}$ Harvard Stem Cell Institute, Boston, MA; \\ ${ }^{3}$ Program in Gastrointestinal Malignancies, Dana-Farber/Harvard Cancer Center, Boston, MA, USA
}

Primary liver cancer is one of the most common cancer worldwide. Hepatocellular carcinoma (HCC) in particular, is the second leading cause of cancer deaths in the world. The Hippo signaling pathway has emerged as a major oncosuppressive pathway that plays critical roles inhibiting hepatocyte proliferation, survival, and HCC formation. A key component of the Hippo pathway is the inhibition of yes-associated protein (YAP)/transcriptional co-activator with PDZ-binding motif (TAZ) transcription factors by the Hippo kinase cascade. Aberrant activation of YAP or TAZ has been found in several human cancers including HCC. It is also well established that YAP/TAZ activation in hepatocytes causes $\mathrm{HCC}$ in mouse models, indicating that YAP/TAZ are potential therapeutic targets for human liver cancer. In this review, we summarize the recent findings regarding the multifarious roles of Hippo/YAP/TAZ in HCC development, and focus on their cell autonomous roles in controlling hepatocyte proliferation, differentiation, survival and metabolism as well as their non-cell autonomous in shaping the tumor microenvironment. (Clin Mol Hepatol 2020;26:742-750)

Keywords: Hippo; YAP/TAZ; Hepatocytes; Liver; Carcinoma, Hepatocellular

\section{INTRODUCTION}

Hepatocellular carcinoma (HCC) is the fifth most common cancer and second leading cause of cancer deaths in the world. ${ }^{1,2}$ It is known that hepatocyte proliferation and survival are tightly controlled by signaling pathways, which also mediate the interactions between hepatocytes and the stroma. Miss-regulation of these signaling pathways led to malignant changes of hepatocytes causing HCC in human and animal models. ${ }^{1,3-8}$ It is imperative to understand how these signaling pathways are regulated and interact with each other.

The Hippo signaling pathway is evolutionarily conserved and plays critical roles restricting cell proliferation and survival in embryonic development and tumorigenesis in adult lives. ${ }^{9,10}$ Key components of this signaling pathway in mammalian cells include a kinase cascade that contains serine/threonine-protein kinase 4/3 (MST1/2, respectively), large tumor suppressor kinases (LATS) $1 / 2$, the transcription coactivators yes-associated protein (YAP)

\footnotetext{
Abbreviations:

CCA, cholangiocarcinoma; cHCC-CCA, combined hepatocellularcholangiocarcinoma; GPCRs, G protein-coupled receptors; HB, hepatoblastoma; HCC, hepatocellular carcinoma; HPCs, hepatic progenitor cells; ICC, intrahepatic cholangiocarcinoma; JAG 1, JAGGED 1; LATS, large tumor suppressor kinases; MCP1, monocyte chemoattractant protein-1; MOB, monopolar spindle-onebinder; NAFLD, nonalcoholic fatty liver disease; PD-L1, programmed cell death ligand 1; SAV1, Salvador family WW domain containing protein 1; $\mathrm{SOH}$, silence of Hippo signaling; TAMs, tumor-associated macrophages; TAZ, transcriptional co-activator with PDZ-binding motif; TEAD, TEA/ATTS domain; TME, tumor microenvironment; Treg, regulatory T cells; WWTR1, WW domain-containing transcription regulator protein 1; YAP, yes-associated protein
}

\section{Corresponding author : Yingzi Yang}

Department of Developmental Biology, Harvard School of Dental Medicine, Harvard Stem Cell Institute, Dana-Farber/Harvard Cancer Center, 188 Longwood Avenue, Boston, MA 02115, USA

Tel: +1-617-432-8304, Fax: +1-617-432-3246

E-mail: yingzi_yang@hsdm.harvard.edu https://orcid.org/0000-0003-3933-887X 
and its paralog WW domain-containing transcription regulator protein 1 (WWTR1; or transcriptional co-activator with PDZ-binding motif [TAZ])." The Hippo pathway receives and is critically regulated by many signaling inputs such as cell contacts, mechanical cues, and G protein-coupled receptors (GPCRs). ${ }^{12-14}$ The Hippo kinase MST1/2 are activated by phosphorylation or trans-autophosphorylation of its activation loop site. MST1/2 bind to its scaffold protein Salvador family WW domain containing protein 1 (SAV1) in a heterotetramer form to facilitate MST1/2 activation and localization to the plasma membrane. Monopolar spindleone-binder (MOB) 1 helps recruit LATS1/2 to MST1/2 to allow LATS phosphorylation by MST1/2 to promote LATS autophosphorylation and activation. The linker phosphorylation sites of MST1/2 also recruit the striatin-interacting phosphatase and kinase complex to dephosphorylate and inactivate MST1/2, therefore creating a negative feedback to restrict MST activity. ${ }^{9,15}$ Upstream regulators such as KIBRA and Mer/NF2 facilitate the Hippo kinase cascade by recruiting LATS to the plasma membrane for its activation by Hippo/MST. ${ }^{9}$ Activated LATS1/2 phosphorylate and inactivate YAP/TAZ subsequently, as phosphorylated YAP and TAZ are sequestered in the cytoplasm by binding to the 14-3-3 protein or are ubiquitinated by $\mathrm{SCF}^{\beta-\mathrm{TRCP}^{-}}$E3 ligase, resulting in proteasomal degradation. ${ }^{16-25}$ When the Hippo kinase cascade is inactivated, YAP and TAZ are not phosphorylated and therefore stabilized and translocate into the nucleus, bind to transcription factors to regulate target gene expression that promotes cell proliferation and survival. ${ }^{26,27}$ TEA/ATTS domain (TEAD) family transcription factors (TEAD1-4) are key partners for YAP/TAZ. ${ }^{27,28}$ The Hippo signaling pathway is oncosuppressive as there are four tumor suppressors intrinsic to this pathway: Hippo kinase MST1/2, LATS1/2, SAV1, and MOB. In the mouse liver, the Hippo kinase MST1 and MST2 inhibit hepatocyte proliferation, survival, and HCC formation by inhibiting YAP/TAZ activation. ${ }^{29-31}$ Many studies have shown that genetically inactivating core components of Hippo pathway leads to development of liver cancer in mice (Table 1).

In the past decade, many studies have established the Hippo signaling pathway as a key regulator of liver growth and tumorigenesis. In mouse liver tumors, the chromosome region of YAP is amplified and nucleus localization of YAP is significantly increased..$^{32}$ Overexpressing activated form of YAP, as well as deleting upstream kinases, leads to hepatomegaly and liver tumorigenesis. ${ }^{16,29-31,33-39}$ In human HCC and cholangiocarcinoma (CCA), the other common type of primary liver cancer that originates from bile duct epithelial cells ${ }^{40}$ abnormal activation of YAP and TAZ has been observed and correlates with high progressive and low differentiated tumor types, resulting in poor prognosis. ${ }^{41-45} \mathrm{Al}$ -

Table 1. Mouse models of Hippo deficient liver

\begin{tabular}{|c|c|c|c|}
\hline Gene & Mouse model & Experimental phenotype and tumor type & Reference \\
\hline \multirow[t]{3}{*}{ YAP } & ApoE/rtTA; TRE-Yap (overexpression) & Hepatomegaly, trabecular type of HCC & 16 \\
\hline & LAP1-tTA; TetOYapS127A (overexpression) & $\begin{array}{l}\text { Hepatomegaly, dysplastic hepatocytes with irregular, enlarged } \\
\text { nuclei, a high nuclear to cytoplasmic ratio, HCC }\end{array}$ & 33 \\
\hline & $\begin{array}{l}\text { AAV-TBG-Cre; R26LSLrtTA; TetOYapS127A } \\
\text { (overexpression) }\end{array}$ & Hepatomegaly, HCC & 34 \\
\hline \multirow[t]{3}{*}{ Mst1/2 } & Ad-Cre; Mst1 ${ }^{-/-}, \mathrm{Mst}^{\mathrm{f} / \mathrm{f}}$ & Hepatomegaly, HCC & 30 \\
\hline & Alb-Cre; Mst1 ${ }^{-/-}, \mathrm{Mst}^{\mathrm{f} /-}$ & Hepatomegaly, HCC/ICC & 29 \\
\hline & Alb-Cre; Mst1 ${ }^{f / f}, M_{s t 2}{ }^{f / f}$ & Hepatomegaly, both well and poorly differentiated HCC, ICC & 25 \\
\hline \multirow[t]{2}{*}{ Nf2 } & Alb-Cre; Nf2 $2^{f / f}$ & Hepatocyte and biliary epithelium over proliferation, HCC/ICC & 37 \\
\hline & Ad-Cre; $N f 2^{f / f}$ & Variety of histopathological types of HCC, poorly differentiated ICC & 38 \\
\hline Sav1 & Alb-Cre; Sav1 $1^{\mathrm{f} / \mathrm{f}}$ & Expansion of hepatic progenitor cells, HCC/CC & 46 \\
\hline Mobla/b & Alb-Cre; Mob1a ${ }^{\mathrm{f} / \mathrm{f}}$, Mob1b $^{-/-}$ & $\begin{array}{l}\text { Hyperplasia of oval cells, hepatocellular adenomas, HCC, ICC or } \\
\text { CHCC-CCA }\end{array}$ & 36 \\
\hline \multirow[t]{2}{*}{ Lats1/2 } & Alb-Cre; Lats1 ${ }^{f / f} ;$ Lats $^{\mathrm{f} / \mathrm{f}}$ & $\begin{array}{l}\text { Expansion of immature BECs, bile duct malformation, perinatal } \\
\text { lethality }\end{array}$ & 49,51 \\
\hline & Ad-Cre; Lats1 $1^{\mathrm{f} / \mathrm{f}} ;$ Lats $2^{\mathrm{f} / \mathrm{f}}$ & Hepatomegaly, biliary cell expansion, ICC & 48 \\
\hline Kibra & Alb-Cre; WWC1 $1^{f / f}, W W C 2^{f / f}$ & $\mathrm{HCC} / \mathrm{ICC}$ & 47 \\
\hline
\end{tabular}

YAP, yes-associated protein; HCC, hepatocellular carcinoma; ICC, intrahepatic cholangiocarcinoma; CHCC-CCA, combined hepatocellular-cholangiocarcinoma; $\mathrm{BEC}$, biliary epithelial cell. 
though inactivation of the oncosuppressive Hippo signaling pathway in the liver promotes tumor formation rapidly, no mutation has been found in core Hippo components in human HCC yet. Recent studies have further identified key function of the Hippo pathway in diverse physiological and pathological processes such as cell fate determination, stem cell regulation, regeneration, and immunity, beyond organ size control in development. ${ }^{9}$ In this review, we discuss the roles of hepatic Hippo signaling pathway in liver tumorigenesis and progression.

\section{HEPATIC HIPPO SIGNALING INHIBITS TUMORI- GENESIS BY CONTROLLING HEPATOCYTE PROLIFERATION AND FATE REPROGRAMMING}

The roles of hepatic Hippo signaling components in liver size control and tumor formation have been extensively studied in mouse mutants (Table 1). Inactivation of Sav1, ${ }^{31,46} \mathrm{Nf}_{2}{ }^{37} \mathrm{Kibra}_{1}{ }^{47}$ Mst1/2, ${ }^{29-31}$ Mobla/b, ${ }^{36}$ or Lats $1 / 2^{48,49}$ results in hepatomegaly and tumor formation. YAP/TAZ are activated in these tumors and liverspecific overexpression of $Y A P^{16,33}$ results in liver tumor formation too. Furthermore, the Hippo-deficient liver tumors can be rescued by reducing YAP/TAZ levels, ${ }^{37,50}$ indicating that YAP/TAZ activation mediates the tumorigenic effects. Although enhanced cell proliferation and survival have been observed in these Hippo-deficient liver tumors, change of hepatocyte fate has also been found, which ultimately determines the liver tumor types. For instance, while Mst1/2 loss driven by the Alb-Cre in mice led to both HCC and intrahepatic cholangiocarcinoma (ICC) formation, ${ }^{29-31} \mathrm{Nf2-}$ or Lats1/2-deficient livers develop liver tumors with both HCC and ICC features (Table 1) ${ }^{37,48,49,51}$ It is possible that the differences are due to different levels of YAP/TAZ activation, but the underlying molecular mechanisms are still not clear. Indeed, it has been found that in human HCC and CCA, combined HCC-CCA and CCA patients showed high expression levels for YAP and TAZ, while only some patients of the HCC group were positive. ${ }^{52}$ In addition, when challenging diagnosis of combined HCC-CCA (CHCC-CCA) was helped with markers of Hippo components, positivity for YAP and TAZ was observed in the hepatocellular and cholangiocellular components of CHCC-CCA, and suggests a single cell origin in combined HCC-CCA. K19 is a specific marker for cholangiocytes. Within the K19- HCC group, YAP and TAZ expression are statistically significant predictor of poor prognosis. ${ }^{52}$

It has been shown that induced YAP activation in mouse hepatocytes leads to hepatocyte dedifferentiate to hepatic progenitor cells (HPCs), which are clonogenic progenitor/stem cells that can differentiate into both hepatocytes and cholangiocytes. ${ }^{34}$ High YAP levels further push the HPCs to become cholangiocytes as termination of YAP activation leads to restoration of hepatocyte fate of the HPCs. ${ }^{34}$ Consistent with findings in mouse models, transcriptome analyses of human liver cancers showed that $\mathrm{HCC}$ patients with the silence of Hippo signaling $(\mathrm{SOH})$ signature had a significantly poorer prognosis than those without the $\mathrm{SOH}$ signature. In addition, there was a significant concordance between the SOH HCC subtype and the hepatic stem cell HCC subtype. ${ }^{43}$ It has been reported that $5-10 \%$ of human HCC has genomic amplification of the genomic locus containing $\mathrm{YAP}^{32}$ and high levels of YAP protein expression have also been detected in a majority of human liver cancers ${ }^{44,53}$ supporting that increased YAP/TAZ activities contribute to liver tumorigenesis. Treatment for advanced HCC in mice using small interfering RNA targeting Yap restores hepatocyte differentiation, leading to tumor regression. ${ }^{54}$

One way for hepatic Hippo signaling to control cell proliferation and differentiation is by interacting with other transcription factors and oncogenic signaling pathways. ${ }^{55-58}$ Among these, activation of Notch, Wnt/ $\beta$-catenin, and Stat3 signaling pathways have been found to cause liver cancers. ${ }^{59-64}$ The Notch signaling pathway has been identified as a functional target of YAP. In human HCC, a Notch ligand JAGGED 1 (JAG 1) has been identified as a direct YAP downstream target contributes to high Notch signaling activity in tumor. ${ }^{65}$ In mice, Notch ligand JAG 1 and Notch receptor Notch2 have been also identified as downstream targets of YAP. ${ }^{34,56}$ Removing Rbpj, which encodes a core transcriptional factor of Notch signaling pathway, or Jag 1 in hepatocytes or inhibiting Notch signaling pharmacologically with DAPT, largely blocked liver tumor formation and hepatocyte to cholangiocyte transdifferentiation. ${ }^{34,56}$ The positive feedback between Notch signaling and YAP/TAZ suggests that Notch signaling could be a therapeutic target for treating HCCS caused by Hippo signaling inactivation or YAP/TAZ activation. Crosstalk between the Hippo/YAP/TAZ and Wnt/B-catenin signaling pathway in HCC and control of hepatocyte fate are complex. In mice, $\beta$-catenin activation and inactivation both promote HCC formation, and $\beta$-catenin activation has been found in liver tumors caused by Mst1/2 deletion. ${ }^{29}$ Coactivation of $\beta$-catenin and YAP was also found in Human hepatoblastoma (HB) —a rare and severe less-differentiated malignant liver tumor, but not in most human HCC or CC. Co-expressing activated forms of $\beta$-catenin and YAP together in mouse hepatocytes induced severe HB-like tumor, which was first found in the central vein area. ${ }^{44}$ While all these studies argue for possible synergistic 
interactions between $\beta$-catenin and YAP during HCC formation, $\beta$-catenin deletion in the Mst1/2 double knock-out mouse liver also significantly promoted initiation and progression of liver tumors formed by hepatocyte-derived HPCs, often found in the portal vein area. ${ }^{56}$ These findings suggest that $\beta$-catenin may play dual roles in regulating YAP activities and cell fate determination. In the peri-central vein area of the liver, Notch signaling activity is low, high $\beta$-catenin levels in hepatocytes coordinate with Yap to promote HB formation under pathological conditions, but in the peri-portal vein area where Notch signaling is higher and HPCS are found, $\beta$-catenin in hepatocytes maintains hepatocyte fate and suppresses hepatocyte dedifferentiation or transdifferentiation by inhibiting Notch signaling activation.

In addition to regulating hepatocyte fate in the liver, Hippo signaling also regulates hepatocyte survival. In the liver-specific Mst1/2 conditional double knock-out mice, hepatocytes are resistant to tumor necrosis factor a-induced apoptosis and acetaminophen-induced hepatotoxicity. ${ }^{29,66}$ Further studies showed that Yap promoted degradation of proapoptotic factors Fox01/3 through Skp2-induced acetylation, ${ }^{67}$ YAP also induced chromosome instability by interacting with FoxM1 ${ }^{45}$ These studies suggest that YAP may promote tumorigenesis by restricting cell death and promoting chromosome instability.

\section{THE HIPPO-YAP SIGNALING PATHWAY REGU- LATES TUMOR MICROENVIRONMENT (TME)}

Since Virchow hypothesized that cancer originated at sites of chronic inflammation in $1863{ }^{68}$ recent studies have expanded the concept that inflammation is a critical component of tumor initiation and progression. It is now clear that TME largely orchestrated by inflammatory cells, is an indispensable participant in neoplastic process, fostering proliferation, survival, and migration. In the last few decades, immunotherapy has shed new light in and become an important part of treating some devastating cancers. Therefore, there is an urgent need to better understand cancer immune interactions, particular under specific contexts of cells, tissues, and molecular pathways involved. Human HCC is an example of inflammation-induced cancer. Chronic viral hepatitis, metabolic liver diseases, and alcohol abuse cause chronic inflammation, which in turn, induces fibrosis, cirrhosis, and cancer. ${ }^{69-71}$ Specifically, macrophages function in the initiation and maintenance of inflammation and tumor-associated macrophages (TAMs) play critical roles during cancer progression. ${ }^{72,73}$
The oncosuppressive Hippo signaling pathway not only plays critical roles inhibiting hepatocyte proliferation, survival, and HCC formation, ${ }^{29-31}$ its activity in hepatocytes also shapes the immune microenvironment by regulating macrophage infiltration and TAM differentiation. ${ }^{29,56}$ We and others recently showed that loss of Hippo kinases MST1/2 or YAP activation in hepatocytes significantly enhanced macrophages infiltration through YAP-dependent monocyte chemoattractant protein-1 (MCP1) expression. ${ }^{50,74}$ MCP1 deletion dramatically reduced the hepatomegaly and tumor formation in the liver specific Mst1/2 double knock-out mice, demonstrating that infiltrated macrophages are critical for initiation and progression of liver tumor. The recruited macrophages further protect the Yap-high tumor initiating cells from immune clearance to promote tumorigenesis. ${ }^{74}$ Related to these findings, TAZ activity in hepatocytes mediates macrophage accumulation in the inflamed and fibrotic liver parenchyma in mouse models of nonalcoholic steatohepatitis, ${ }^{75}$ which often precedes HCC.

Programmed cell death ligand 1 (PD-L1) is an immune checkpoint molecule that inhibits cytotoxic $\mathrm{T}$ cell function to allow tumor cells evade immune surveillance. Interestingly, PD-L1 is a target of Hippo signaling, and TAZ-induced PD-L1 upregulation was sufficient to inhibit the function of cytotoxic T cells. ${ }^{76}$ Moreover, tumor-derived lactate also induced PD-L1 expression in a TAZ-dependent manner. ${ }^{76,77}$ However, clinical studies for HCC revealed that the efficacy of blocking PD-L1 is not significantly better than existing drugs. But recently, more encouraging results showed that combination of atezolizumab (anti-PD-L1) and bevacizumab (anti-vascular endothelial-derived growth factor) had better overall and progression-free survival than sorafenib in treating unresectable HCC (IMbrave 150). ${ }^{78}$ In addition, Moroishi et al. ${ }^{79}$ also observed that tumor cells with deleted Lats1/2, while activated YAP/TAZ, significantly boosted anti-tumor immune responses and inhibited tumor growth by secreting nucleic-acid-rich extracellular vesicles, which induced a type I interferon response via the Tolllike receptors-MYD88/TRIF pathway. It will be important to further understand the underlying mechanism for the difference.

It is known that a Hippo kinase MST1 plays important roles in human immune system. Mst1 deficiency in human patients caused immunodeficiency and lymphopenia. ${ }^{80}$ Animal studies also showed that Mst1/2 conditional deletion in immune cells results in features of autoimmune phenotypes including lymphocyte infiltration, Th-17 overactivation and autoantibody production. ${ }^{81}$ While TAZ was shown to inhibit regulatory $T$ cells (Treg) differentiation and promote Th-17 differentiation, ${ }^{81}$ YAP was found to be necessary for Treg differentiation and function in the suppression 


\section{CLINICAL and MOLECULAR}

of antitumor immunity by activating activin/SMAD signaling ${ }^{82}$ or through increasing the expression of transforming growth factor $\beta$ receptor- $2 .{ }^{83}$ YAP and TAZ are both downstream effectors of MST1/2, the distinct roles of TAZ and YAP in Treg differentiation indicate the necessity to further investigate their regulations and targets in T cells.

\section{HIPPO SIGNALING PATHWAY IN TUMOR META- BOLIC REPROGRAMMING}

As Hippo signaling critically controls cell proliferation and survival, it is not surprising that this pathway both regulates and is regulated by cell metabolism and energy availability. YAP and TAZ have emerged as nodes in coordinating nutrient and energy production and expenditure. While high levels of glucose and fatty acids activate YAP/TAZ transcription, YAP/TAZ have also been shown to promote metabolic processes such as glycolysis, lipogenesis, and glutaminolysis to ensure adequate nutrient supply for cell and tissue growth. ${ }^{84}$ Aerobic glycolysis has been recognized as a common hallmark of solid tumors. Glycolytic metabolism provides the majority of chemical precursors required for the synthesis of nucleotides, amino acids, and lipids which are necessary building blocks for cell growth and proliferation. In the liver, excess nutrient supply can lead to steatosis and steatohepatitis, conditions called nonalcoholic fatty liver disease (NAFLD), in which abnormal lipid accumulation results in inflammation and death of hepatocytes, promoting fibrosis, cirrhosis, and eventually liver cancer. In mouse NAFLD models and in human steatohepatitis samples, increased YAP/TAZ levels have been observed, associated with ductular reactions. It has been reported that expression of activated TAZ promotes inflammation, NAFLD, and tumor formation, linking TAZ - but not YAP, to NAFLD, but not steatosis, in tumor development. ${ }^{75,85}$ One mechanism how steatosis could promote YAP/TAZ activation is through the obesity-associated protein JCAD that inhibits upstream LATS2 ${ }^{86}$ The differential roles of YAP and TAZ have been reported under multiple context including NAFLD, it will be important to further understand the molecular mechanisms that determine the functional specificity of YAP and TAZ.

Glycolytic metabolism in tumor cells also plays crucial roles in rewriting the TME, and facilitates the tumor growth, angiogenesis, metastasis, as well as immune surveillance. For example, lactate, the metabolite of glycolysis could directly trigger M2 polarization of the macrophages; suppress the proliferation and cytotoxic function of T cells. Recent studies showed that aerobic glycolysis increased YAP/TAZ transcriptional activity through the interaction with PFK1 or AMPK inhibition. ${ }^{87}$ On the other hand,

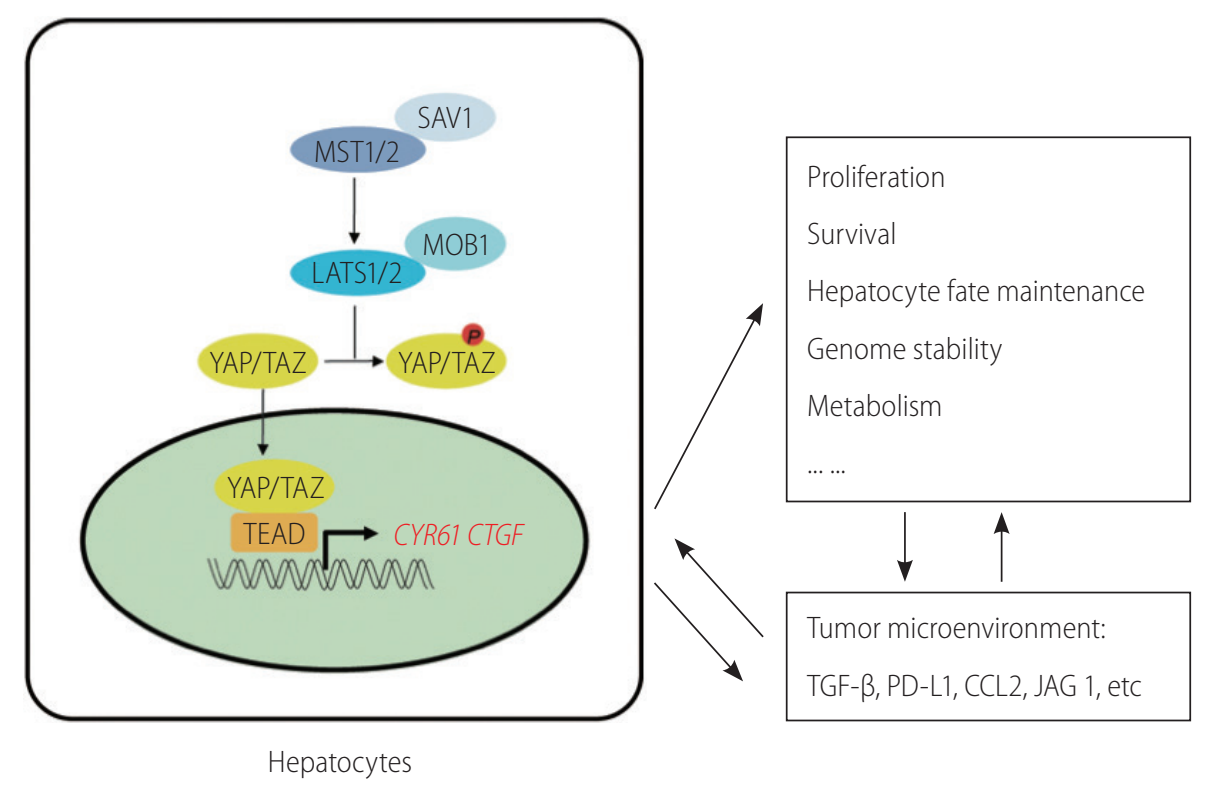

Figure 1. Schematics of the roles of Hippo signaling in hepatocytes. Hippo signaling plays multiple roles in hepatocytes, and these functions may all shape tumor microenvironment if tumor arises as a result of Hippo signaling deficiency. In addition, Hippo signaling activities are intimately regulated by the microenvironment surrounding the hepatocytes. SAV1, Salvador family WW domain containing protein 1; LATS, large tumor suppressor kinases; YAP, yes-associated protein; PD-L1, programmed cell death ligand 1; JAG 1, JAGGED 1. 
glucose starvation or the glycolysis inhibitor 2-deoxy-D-glucoseinduced cellular energy stress, which increased AMPK-dependent LATS activation that inhibits YAP/TAZ activation. ${ }^{88}$ In addition, administration of methylglyoxal, a metabolite of glycolysis, increases YAP activation and nuclear translocation by increasing heat shock protein 90 glycation and LATS1 degradation. ${ }^{89}$

YAP/TAZ activation also regulates the cellular metabolic reprogramming in tumor cells. Hypoxia is a well-known common feature in most solid TME. A recent study revealed that hypoxic stress promoted YAP/HIF1A interaction and PKM2 expression to further increase glycolysis in HCC cells. ${ }^{90}$ In addition, HMGB1 was found to increase glycolysis and liver tumorigenesis in a YAP/HIF1-a dependent manner, and inhibition of the HMGB1-YAP-HIF1-a pathway reduced glycolysis and liver tumor growth. ${ }^{91}$ The mevalonate pathway is responsible for production of cholesterol, bile acids and steroid hormones and is often upregulated in liver tumor. It was shown that the mevalonate pathway is critical for YAP/TAZ activity and the underlying mechanism is mediated by RHO membrane localization and activity. ${ }^{92}$ Inhibiting the mevalonate pathway by statins significantly suppressed TAZ levels and improved the prognosis of HCC patients. ${ }^{93}$ Therefore, the mevalonate pathway could be a therapeutic target to reduce YAP/TAZ activation for liver tumor treatment. Taken together, these findings indicate that Hippo/YAP signaling can also control tumor initiation and progression by regulating cell metabolism.

\section{CONCLUSION AND FUTURE PERSPECTIVES}

The hepatocyte appears to be a major cell type that is regulated by the Hippo signaling pathway in multiple ways (Fig. 1). As our knowledge about how Hippo signaling is transduced and regulated in hepatocytes expands over the last decade, the molecular mechanisms whereby Hippo signaling regulates multiple aspects hepatocyte physiology and pathology remain to be further elucidated. For example, although YAP/TAZ promote hepatocyte proliferation and survival, YAP/TAZ-deficient liver can still regenerate. In addition, in acute and chronic liver injury, it will be important to understand how Hippo signaling regulates HPC formation and hepatocyte/cholangiocyte fate determination. Hippo signaling interacts with many signaling pathways and therefore, when considering the Hippo pathway as therapeutic targets, its pleiotropic functions must be carefully considered. While inhibition of YAP or TAZ is beneficial to reducing steatohepatitis, cirrhosis, and cancer, it could inhibit liver regeneration upon liver cell damage. In addi- tion, it is still a mystery in several cases why YAP and TAZ appear to differentially regulate a similar process. Finally, because of the proved roles of Hippo signaling in regulating factors important in TME (Fig. 1), and the success of immune therapy, blocking Hippo signaling in mediating reciprocal interactions between pre-tumor/ tumor cells and TME may provide promising HCC/ICC therapeutic strategies. However, there are many unanswered fundamental questions regarding hepatic function of Hippo signaling in regulating TME and TAM differentiation. 1) What are the populations of the TAMs and how are they induced by Hippo-deficient hepatocytes? 2) What are the other immune cells in the TME and how are they regulated by hepatic Hippo signaling? The knowledge gained by answering these questions will establish a solid new foundation for further mechanistic investigation of hepatic Hippo signaling in inducing inflammation, TME remodeling and provide new targets and strategies to treat HCC.

\section{Authors' contribution}

Yuchen Liu and Xiaohui Wang wrote the first draft. Yingzi Yang revised and edited the manuscript.

\section{Acknowledgements}

This work is support by NCl grant R01CA222571.

\section{Conflicts of Interest}

The authors have no conflicts to disclose.

\section{REFERENCES}

1. El-Serag HB. Hepatocellular carcinoma. N Engl J Med 2011;365:11181127

2. Torre LA, Bray F, Siegel RL, Ferlay J, Lortet-Tieulent J, Jemal $A$. Global cancer statistics, 2012. CA Cancer J Clin 2015;65:87-108.

3. Farazi PA, DePinho RA. Hepatocellular carcinoma pathogenesis: from genes to environment. Nat Rev Cancer 2006;6:674-687.

4. Hanahan D, Weinberg RA. Hallmarks of cancer: the next generation. Cell 2011;144:646-674.

5. Forner A, Llovet JM, Bruix J. Hepatocellular carcinoma. Lancet 2012;379:1245-1255.

6. Yu $H$, Lee $H$, Herrmann A, Buettner R, Jove R. Revisiting STAT3 signalling in cancer: new and unexpected biological functions. Nat Rev Cancer 2014;14:736-746.

7. Miyajima A, Tanaka M, Itoh T. Stem/progenitor cells in liver development, homeostasis, regeneration, and reprogramming. Cell Stem Cell 2014;14:561-574. 
8. Tahmasebi Birgani M, Carloni V. Tumor microenvironment, a paradigm in hepatocellular carcinoma progression and therapy. Int J Mol Sci 2017;18:405.

9. Zheng Y, Pan D. The hippo signaling pathway in development and disease. Dev Cell 2019;50:264-282.

10. Yu FX, Zhao B, Guan KL. Hippo pathway in organ size control, tissue homeostasis, and cancer. Cell 2015;163:811-828.

11. Pan D. The hippo signaling pathway in development and cancer. Dev Cell 2010;19:491-505.

12. Aragona M, Panciera T, Manfrin A, Giulitti S, Michielin F, Elvassore N, et al. A mechanical checkpoint controls multicellular growth through YAP/TAZ regulation by actin-processing factors. Cell 2013;154:10471059.

13. Dupont S, Morsut L, Aragona M, Enzo E, Giulitti S, Cordenonsi M, et al. Role of YAP/TAZ in mechanotransduction. Nature 2011;474:179183.

14. Yu FX, Zhao B, Panupinthu N, Jewell JL, Lian I, Wang LH, et al. Regulation of the hippo-YAP pathway by G-protein-coupled receptor signaling. Cell 2012;150:780-791.

15. Chen R, Xie R, Meng Z, Ma S, Guan KL. STRIPAK integrates upstream signals to initiate the hippo kinase cascade. Nat Cell Biol 2019;21:1565-1577.

16. Dong J, Feldmann G, Huang J, Wu S, Zhang N, Comerford SA, et al. Elucidation of a universal size-control mechanism in Drosophila and mammals. Cell 2007;130:1120-1133.

17. Zhao B, Wei X, Li W, Udan RS, Yang Q, Kim J, et al. Inactivation of YAP oncoprotein by the hippo pathway is involved in cell contact inhibition and tissue growth control. Genes Dev 2007;21:2747-2761.

18. Zhao B, Li L, Tumaneng K, Wang CY, Guan KL. A coordinated phosphorylation by Lats and CK1 regulates YAP stability through SCF(beta-TRCP). Genes Dev 2010;24:72-85.

19. Kwon Y, Vinayagam A, Sun $X$, Dephoure N, Gygi SP, Hong $P$, et al. The hippo signaling pathway interactome. Science 2013;342:737740.

20. Callus BA, Verhagen AM, Vaux DL. Association of mammalian sterile twenty kinases, Mst1 and Mst2, with hSalvador via C-terminal coiled-coil domains, leads to its stabilization and phosphorylation. FEBS J 2006;273:4264-4276.

21. Praskova M, Xia F, Avruch J. MOBKL1A/MOBKL1B phosphorylation by MST1 and MST2 inhibits cell proliferation. Curr Biol 2008;18:311321.

22. Tapon N, Harvey KF, Bell DW, Wahrer DC, Schiripo TA, Haber D, et al. Salvador promotes both cell cycle exit and apoptosis in drosophila and is mutated in human cancer cell lines. Cell 2002;110:467478.

23. Wu S, Huang J, Dong J, Pan D. Hippo encodes a Ste-20 family protein kinase that restricts cell proliferation and promotes apoptosis in conjunction with salvador and warts. Cell 2003;114:445-456.
24. Hao Y, Chun A, Cheung K, Rashidi B, Yang X. Tumor suppressor LATS1 is a negative regulator of oncogene YAP. J Biol Chem 2008;283:5496-5509.

25. Liu CY, Zha ZY, Zhou X, Zhang H, Huang W, Zhao D, et al. The hippo tumor pathway promotes TAZ degradation by phosphorylating a phosphodegron and recruiting the SCF $\beta$-TrCP E3 ligase. J Biol Chem 2010;285:37159-37169.

26. Lai $D$, Ho KC, Hao Y, Yang $X$. Taxol resistance in breast cancer cells is mediated by the hippo pathway component TAZ and its downstream transcriptional targets Cyr61 and CTGF. Cancer Res 2011;71:27282738.

27. Zhao B, Ye X, Yu J, Li L, Li W, Li S, et al. TEAD mediates YAP-dependent gene induction and growth control. Genes Dev 2008;22:19621971.

28. Vassilev A, Kaneko KJ, Shu H, Zhao Y, DePamphilis ML. TEAD/ TEF transcription factors utilize the activation domain of YAP65, a src/yes-associated protein localized in the cytoplasm. Genes Dev 2001;15:1229-1241.

29. Song H, Mak KK, Topol L, Yun K, Hu J, Garrett L, et al. Mammalian Mst1 and Mst2 kinases play essential roles in organ size control and tumor suppression. Proc Natl Acad Sci U S A 2010;107:1431-1436.

30. Zhou D, Conrad C, Xia F, Park JS, Payer B, Yin Y, et al. Mst1 and Mst2 maintain hepatocyte quiescence and suppress hepatocellular carcinoma development through inactivation of the Yap1 oncogene. Cancer Cell 2009;16:425-438.

31. Lu L, Li Y, Kim SM, Bossuyt W, Liu P, Qiu Q, et al. Hippo signaling is a potent in vivo growth and tumor suppressor pathway in the mammalian liver. Proc Natl Acad Sci U S A 2010;107:1437-1442.

32. Zender L, Spector MS, Xue W, Flemming P, Cordon-Cardo C, Silke J, et al. Identification and validation of oncogenes in liver cancer using an integrative oncogenomic approach. Cell 2006;125:1253-1267.

33. Camargo FD, Gokhale S, Johnnidis JB, Fu D, Bell GW, Jaenisch R, et al. YAP1 increases organ size and expands undifferentiated progenitor cells. Curr Biol 2007;17:2054-2060.

34. Yimlamai D, Christodoulou C, Galli GG, Yanger K, Pepe-Mooney B, Gurung $B$, et al. Hippo pathway activity influences liver cell fate. Cell 2014;157:1324-1338.

35. Nishio M, Hamada K, Kawahara K, Sasaki M, Noguchi F, Chiba S, et al. Cancer susceptibility and embryonic lethality in Mob1a/1b double-mutant mice. J Clin Invest 2012;122:4505-4518.

36. Nishio M, Sugimachi K, Goto H, Wang J, Morikawa T, Miyachi Y, et al. Dysregulated YAP1/TAZ and TGF- $\beta$ signaling mediate hepatocarcinogenesis in Mob1a/1b-deficient mice. Proc Natl Acad Sci U S A 2016;113:E71-E80.

37. Zhang N, Bai H, David KK, Dong J, Zheng Y, Cai J, et al. The Merlin/ NF2 tumor suppressor functions through the YAP oncoprotein to regulate tissue homeostasis in mammals. Dev Cell 2010;19:27-38.

38. Benhamouche S, Curto M, Saotome I, Gladden AB, Liu CH, Giovan- 
nini $\mathrm{M}$, et al. Nf2/Merlin controls progenitor homeostasis and tumorigenesis in the liver. Genes Dev 2010;24:1718-1730.

39. Yin F, Yu J, Zheng Y, Chen Q, Zhang N, Pan D. Spatial organization of hippo signaling at the plasma membrane mediated by the tumor suppressor Merlin/NF2. Cell 2013;154:1342-1355.

40. Khan SA, Thomas HC, Davidson BR, Taylor-Robinson SD. Cholangiocarcinoma. Lancet 2005;366:1303-1314.

41. Guo Y, Pan Q, Zhang J, Xu X, Liu X, Wang Q, et al. Functional and clinical evidence that TAZ is a candidate oncogene in hepatocellular carcinoma. J Cell Biochem 2015;116:2465-2475.

42. Xu MZ, Yao TJ, Lee NP, Ng IO, Chan YT, Zender L, et al. Yes-associated protein is an independent prognostic marker in hepatocellular carcinoma. Cancer 2009;115:4576-4585.

43. Sohn BH, Shim JJ, Kim SB, Jang KY, Kim SM, Kim JH, et al. Inactivation of hippo pathway is significantly associated with poor prognosis in hepatocellular carcinoma. Clin Cancer Res 2016;22:1256-1264.

44. Tao J, Calvisi DF, Ranganathan S, Cigliano A, Zhou L, Singh S, et al. Activation of $\beta$-catenin and Yap1 in human hepatoblastoma and induction of hepatocarcinogenesis in mice. Gastroenterology 2014; 147:690-701.

45. Weiler SME, Pinna F, Wolf T, Lutz T, Geldiyev A, Sticht C, et al. Induction of chromosome instability by activation of yes-associated protein and forkhead box M1 in liver cancer. Gastroenterology 2017;152:2037-2051.e22.

46. Lee KP, Lee JH, Kim TS, Kim TH, Park HD, Byun JS, et al. The hipposalvador pathway restrains hepatic oval cell proliferation, liver size, and liver tumorigenesis. Proc Natl Acad Sci U S A 2010;107:82488253.

47. Hermann A, Wennmann DO, Gromnitza S, Edeling M, Van Marck $V$, Sudol $M$, et al. WW and C2 domain-containing proteins regulate hepatic cell differentiation and tumorigenesis through the hippo signaling pathway. Hepatology 2018;67:1546-1559.

48. Chen Q, Zhang N, Xie R, Wang W, Cai J, Choi KS, et al. Homeostatic control of Hippo signaling activity revealed by an endogenous activating mutation in YAP. Genes Dev 2015;29:1285-1297.

49. Yi J, Lu L, Yanger K, Wang W, Sohn BH, Stanger BZ, et al. Large tumor suppressor homologs 1 and 2 regulate mouse liver progenitor cell proliferation and maturation through antagonism of the coactivators YAP and TAZ. Hepatology 2016;64:1757-1772.

50. Kim W, Khan SK, Liu Y, Xu R, Park O, He Y, et al. Hepatic hippo signaling inhibits protumoural microenvironment to suppress hepatocellular carcinoma. Gut 2018;67:1692-1703.

51. Lee DH, Park JO, Kim TS, Kim SK, Kim TH, Kim MC, et al. LATSYAP/TAZ controls lineage specification by regulating TGF $\beta$ signaling and Hnf4a expression during liver development. Nat Commun 2016;7:11961.

52. Van Haele M, Moya IM, Karaman R, Rens G, Snoeck J, Govaere O, et al. YAP and TAZ heterogeneity in primary liver cancer: an analysis of its prognostic and diagnostic role. Int J Mol Sci 2019;20:638.

53. Li H, Wolfe A, Septer S, Edwards G, Zhong X, Abdulkarim AB, et al. Deregulation of hippo kinase signalling in human hepatic malignancies. Liver Int 2012;32:38-47.

54. Fitamant J, Kottakis F, Benhamouche S, Tian HS, Chuvin N, Parachoniak $C A$, et al. YAP inhibition restores hepatocyte differentiation in advanced hcc, leading to tumor regression. Cell Rep 2015;10:16921707.

55. Zhang S, Zhou D. Role of the transcriptional coactivators YAP/TAZ in liver cancer. Curr Opin Cell Biol 2019;61:64-71.

56. Kim W, Khan SK, Gvozdenovic-Jeremic J, Kim Y, Dahlman J, Kim H, et al. Hippo signaling interactions with Wnt/B-catenin and Notch signaling repress liver tumorigenesis. J Clin Invest 2017;127:137-152.

57. Kim W, Cho YS, Wang X, Park O, Ma X, Kim H, et al. Hippo signaling is intrinsically regulated during cell cycle progression by APC/CCdh1. Proc Natl Acad Sci U S A 2019;116:9423-9432.

58. Cho YS, Li S, Wang X, Zhu J, Zhuo S, Han Y, et al. CDK7 regulates organ size and tumor growth by safeguarding the hippo pathway effector Yki/Yap/Taz in the nucleus. Genes Dev 2020;34:53-71.

59. de La Coste A, Romagnolo B, Billuart $P$, Renard CA, Buendia MA, Soubrane 0 , et al. Somatic mutations of the beta-catenin gene are frequent in mouse and human hepatocellular carcinomas. Proc Natl Acad Sci U S A 1998;95:8847-8851.

60. He G, Yu GY, Temkin V, Ogata H, Kuntzen C, Sakurai T, et al. Hepatocyte IKKbeta/NF-kappaB inhibits tumor promotion and progression by preventing oxidative stress-driven STAT3 activation. Cancer Cell 2010;17:286-297.

61. He G, Karin M. NF-kB and STAT3 - key players in liver inflammation and cancer. Cell Res 2011;21:159-168.

62. Villanueva A, Alsinet C, Yanger K, Hoshida Y, Zong Y, Toffanin S, et al. Notch signaling is activated in human hepatocellular carcinoma and induces tumor formation in mice. Gastroenterology 2012;143: 1660-1669.e7.

63. Zong Y, Panikkar A, Xu J, Antoniou A, Raynaud P, Lemaigre F, et al. Notch signaling controls liver development by regulating biliary differentiation. Development 2009;136:1727-1739.

64. Geisler F, Strazzabosco M. Emerging roles of Notch signaling in liver disease. Hepatology 2015;61:382-392.

65. Tschaharganeh DF, Chen X, Latzko P, Malz M, Gaida MM, Felix K, et al. Yes-associated protein up-regulates Jagged-1 and activates the Notch pathway in human hepatocellular carcinoma. Gastroenterology 2013;144:1530-1542.e12.

66. Wu H, Xiao Y, Zhang S, Ji S, Wei L, Fan F, et al. The Ets transcription factor GABP is a component of the hippo pathway essential for growth and antioxidant defense. Cell Rep 2013;3:1663-1677.

67. Zhang S, Chen Q, Liu Q, Li Y, Sun X, Hong L, et al. Hippo signaling suppresses cell ploidy and tumorigenesis through Skp2. Cancer Cell 2017;31:669-684.e7. 
68. Balkwill F, Mantovani A. Inflammation and cancer: back to Virchow? Lancet 2001;357:539-545.

69. Block TM, Mehta AS, Fimmel CJ, Jordan R. Molecular viral oncology of hepatocellular carcinoma. Oncogene 2003;22:5093-5107.

70. Canavese M, Wijesundara D, Maddern GJ, Grubor-Bauk B, Hauben E. Hepatitis $C$ virus drives the pathogenesis of hepatocellular carcinoma: from immune evasion to carcinogenesis. Clin Transl Immunology 2016;5:e101.

71. Nakamoto Y, Guidotti LG, Kuhlen CV, Fowler P, Chisari FV. Immune pathogenesis of hepatocellular carcinoma. J Exp Med 1998;188:341350.

72. Wynn TA, Chawla A, Pollard JW. Macrophage biology in development, homeostasis and disease. Nature 2013;496:445-455.

73. Noy R, Pollard JW. Tumor-associated macrophages: from mechanisms to therapy. Immunity 2014;41:49-61.

74. Guo X, Zhao Y, Yan H, Yang Y, Shen S, Dai X, et al. Single tumorinitiating cells evade immune clearance by recruiting type II macrophages. Genes Dev 2017;31:247-259.

75. Wang X, Zheng Z, Caviglia JM, Corey KE, Herfel TM, Cai B, et al. Hepatocyte TAZ/WWTR1 promotes inflammation and fibrosis in nonalcoholic steatohepatitis. Cell Metab 2016;24:848-862.

76. Janse van Rensburg HJ, Azad T, Ling M, Hao Y, Snetsinger B, Khanal $P$, et al. The hippo pathway component TAZ promotes immune evasion in human cancer through PD-L1. Cancer Res 2018;78:14571470.

77. Feng J, Yang H, Zhang Y, Wei H, Zhu Z, Zhu B, et al. Tumor cell-derived lactate induces TAZ-dependent upregulation of PD-L1 through GPR81 in human lung cancer cells. Oncogene 2017;36:5829-5839.

78. Finn RS, Qin S, Ikeda M, Galle PR, Ducreux M, Kim TY, et al. Atezolizumab plus bevacizumab in unresectable hepatocellular carcinoma. N Engl J Med 2020;382:1894-1905.

79. Moroishi T, Hayashi T, Pan WW, Fujita Y, Holt MV, Qin J, et al. The hippo pathway kinases LATS1/2 suppress cancer immunity. Cell 2016;167:1525-1539.e17.

80. Abdollahpour H, Appaswamy G, Kotlarz D, Diestelhorst J, Beier R, Schäffer $A A$, et al. The phenotype of human STK4 deficiency. Blood 2012;119:3450-3457.

81. Geng J, Yu S, Zhao H, Sun X, Li X, Wang P, et al. The transcriptional coactivator TAZ regulates reciprocal differentiation of TH17 cells and
Treg cells. Nat Immunol 2017;18:800-812.

82. Ni X, Tao J, Barbi J, Chen Q, Park BV, Li Z, et al. YAP is essential for treg-mediated suppression of antitumor immunity. Cancer Discov 2018;8:1026-1043.

83. Fan Y, Gao Y, Rao J, Wang K, Zhang F, Zhang C. YAP-1 promotes tregs differentiation in hepatocellular carcinoma by enhancing TGFBR2 transcription. Cell Physiol Biochem 2017;41:1189-1198.

84. Koo JH, Guan KL. Interplay between YAP/TAZ and metabolism. Cell Metab 2018;28:196-206.

85. Hagenbeek TJ, Webster JD, Kljavin NM, Chang MT, Pham T, Lee HJ, et al. The hippo pathway effector TAZ induces TEAD-dependent liver inflammation and tumors. Sci Signal 2018;11:eaaj1757.

86. Ye J, Li TS, Xu G, Zhao YM, Zhang NP, Fan J, et al. JCAD promotes progression of nonalcoholic steatohepatitis to liver cancer by inhibiting LATS2 kinase activity. Cancer Res 2017;77:5287-5300.

87. Enzo E, Santinon G, Pocaterra A, Aragona M, Bresolin S, Forcato $M$, et al. Aerobic glycolysis tunes YAP/TAZ transcriptional activity. EMBO J 2015;34:1349-1370.

88. Mo JS, Meng Z, Kim YC, Park HW, Hansen CG, Kim S, et al. Cellular energy stress induces AMPK-mediated regulation of YAP and the hippo pathway. Nat Cell Biol 2015;17:500-510.

89. Huntoon CJ, Nye MD, Geng L, Peterson KL, Flatten KS, Haluska P, et al. Heat shock protein 90 inhibition depletes LATS1 and LATS2, two regulators of the mammalian hippo tumor suppressor pathway. Cancer Res 2010;70:8642-8650.

90. Zhang X, Li Y, Ma Y, Yang L, Wang T, Meng X, et al. Yes-associated protein (YAP) binds to HIF-1a and sustains HIF-1a protein stability to promote hepatocellular carcinoma cell glycolysis under hypoxic stress. J Exp Clin Cancer Res 2018;37:216.

91. Chen R, Zhu S, Fan XG, Wang H, Lotze MT, Zeh HJ 3rd, et al. High mobility group protein $\mathrm{B} 1$ controls liver cancer initiation through yes-associated protein - dependent aerobic glycolysis. Hepatology 2018:67:1823-1841.

92. Sorrentino G, Ruggeri N, Specchia V, Cordenonsi M, Mano M, Dupont $\mathrm{S}$, et al. Metabolic control of YAP and TAZ by the mevalonate pathway. Nat Cell Biol 2014;16:357-366.

93. Higashi T, Hayashi H, Kitano Y, Yamamura K, Kaida T, Arima K, et al. Statin attenuates cell proliferative ability via TAZ (WWTR1) in hepatocellular carcinoma. Med Oncol 2016;33:123. 